\title{
Influence des traitements thermiques sur la perméabilité initiale de l'alliage amorphe $\mathrm{Fe}_{78} \mathrm{Si}_{9} \mathrm{~B}_{13}$
}

\author{
F. ALVES et J.C. PERRON
}

Laboratoire de Génie Electrique de Paris, E.S.E., Plateau du Moulon, 91192 Gif-sur-Yvette cedex, France

\begin{abstract}
Résumé. - La relaxation magnétique de l'alliage $\mathrm{Fe}_{78} \mathrm{Sig}_{9} \mathrm{~B}_{13}$ a été étudiée en fonction de la temperature et en fonction du temps le long des isothermes. En dessous d'une temperature de recuit d'environ $250^{\circ} \mathrm{C}$, les processus de désaccommodation de la perméabilité initiale sont dominants alors qu'au- dessus il s'agit de processus d'accommodation. Dans ce demier cas, on observe une évolution irréversible de l'ordre magnétique.

Abstract. - Magnetic relaxation of $\mathrm{Fe}_{78} \mathrm{Sig}_{13}$ amorphous alloy has been studied as a function of temperature and as a function of time along the isotherms. Below an annealing temperature of $250^{\circ} \mathrm{C}$ disaccommodation are dominant when above this temperature accommodation processes occur. In this later range we observed an irreversible evolution of magnetic order.
\end{abstract}

\section{Introduction.}

Les matériaux magnétiques amorphes à base de Fer, bruts d'élaboration, ne présentent pas un ferromagnétisme doux remarquable. Le mode de préparation (hypertrempe) en est la cause. Les contraintes dues à la vitesse de refroidissement $\left(-10^{6}{ }^{\circ} \mathrm{C} / \mathrm{s}\right)$ induisent des inhomogénéités de structures importantes piégeant le mouvement des parois de Bloch et par conséquent une perméabilité faible et des pertes élevées, préjudiciables pour une utilisation en basse fréquence ( $\mathrm{F} \leq 1 \mathrm{kHz}$ ).

L'intérêt des traitements thermiques est de relaxer ces contraintes " internes ". On observe une évolution de l'état amorphe vers un état dit "relaxé". Cette dernière est mise en évidence par des mesures d'enthalpie obtenues par calorimétrie différentielle à balayage. Lors des recuits, les deux paramètres temps et température sont essentiels. Nous avons étudié les variations temporelles de la perméabilité initiale lors de paliers isothermes à des températures comprises entre l'ambiante et la température de Curie. Il est montré que ces variations sont essentiellement gouvernées par l'anisotropie magnétique locale ( en relation avec l'environnement local des atomes porteurs de moments magnétiques ). Dans la gamme de température explorée, nous observons deux évolutions antagonistes de la perméabilité initiale qui déterminent les propriétés magnétiques finales de l'échantillon à la température ambiante.

\section{Techniques expérimentales.}

Nous rapportons ici les résultats obtenus sur l'alliage $\mathrm{Fe}_{78} \mathrm{SigB}_{13}$ commercialisé par Allied Corporation sous la désignation Metglas 2605 S2. Les échantillons se présentent sous forme de ruban ( largeur $25 \mathrm{~mm}$ épaisseur $20 \mu \mathrm{m}$ ). Pour nos différents essais, une longueur de $2,5 \mathrm{~m}$ de ruban est enroulée sur un support cylindrique de diamètre $38 \mathrm{~mm}$. 
Tous les recuits sont réalisés sous Argon pour empêcher toute oxydation. Le cycle thermique consiste en un palier isotherme de 3 heures à une température de recuit $T_{r}$ avec des vitesses de montée et de descente en température de $10^{\circ} \mathrm{C} / \mathrm{min}$.

Des mesures de chaleur spécifique $C_{p}(T)$ sont réalisées sur un système DSC 4 avec une vitesse de chauffe de $40^{\circ} \mathrm{C} / \mathrm{min}$. Les énergies de relaxation $\Delta H$ sont évaluées et nous pouvons définir un taux de relaxation structurale ( TRS ) par une méthode décrite par ailleurs [1]. Ce dernier correspond à la proportion des défauts annihilés durant le recuit. Les thermogrammes donnent aussi la température de Curie $\left(\mathrm{T}_{\mathrm{c}}\right)$ qui est associée à un phénomène de relaxation endothermique.

La perméabilité à faible champ est mesurée en appliquant un champ d'excitation sinusoïdal d'amplitude constante $\left(\mathrm{F}=1 \mathrm{kHz}, \mathrm{h}_{\mathrm{ac}}<1 \mathrm{~A} / \mathrm{m}\right)$ juste après une démagnétisation à $50 \mathrm{~Hz}$ (décroissance linéaire du champ d'excitation de $100 \mathrm{~A} / \mathrm{m}$ à zéro sur une durée de 2 mins. ).

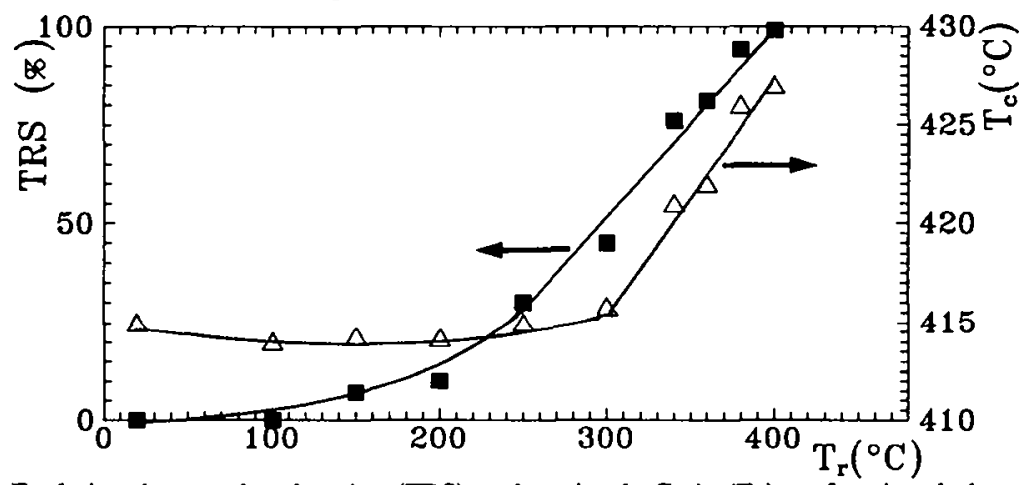

Fig. 1.- Evolution du taux de relaxation (TRS) et du point de Curie $\left(\mathrm{T}_{c}\right)$ en fonction de la temperature de recuit (durée : $3 \mathrm{~h}$ ).

\section{Résultats.}

La grandeur TRS (Fig. 1) représente le taux de relaxation structurale ou degré d'évolution de la structure amorphe vers un état thermodynamique plus stable. Il est défini comme le rapport $\left(\Delta \mathrm{H}_{\mathrm{b}}-\Delta \mathrm{H}_{\mathrm{r}}\right) / \Delta \mathrm{H}_{\mathrm{b}}$ où $\Delta \mathrm{H}_{\mathrm{b}}$ et $\Delta \mathrm{H}_{\mathrm{r}}$ sont respectivement les énergies enthalpiques des échantillons brut de livraison et recuit. Pour une durée de recuit de 3 heures, l'annihilation des défauts internes, qui s'accompagne par une densification de l'alliage amorphe (diminution du volume libre piégé lors de la trempe), n'est effective qu'à partir de $\mathrm{T}_{\mathrm{T}}$ de l'ordre de $200^{\circ} \mathrm{C}$. L'évolution de la température de Curie, qui traduit l'ordre magnétique donc le couplage entre les moments magnétiques, n'est observable seulement qu'à partir de $T_{r}$ voisine de $300{ }^{\circ} \mathrm{C}$ environ. Nous observons que l'évolution de l'ordre magnétique intervient après une évolution de la structure de l'ordre de $40 \%$ par le biais de réarrangements de type topologique probablement. Il est à noter que, contrairement aux alliages à base Cobalt $(\mathrm{Ms}=0.55 \mathrm{~T})$, les énergies $\Delta H$ sont notables du fait de l'existence d'interactions magnétiques fortes entre les atomes de Fer.

La figure 2 représente l'évolution de la perméabilité initiale en fonction de la température pour un échantillon brut de trempe dans les condition suivantes : $F=1 \mathrm{kHz}, h_{\mathrm{ac}}=0,1 \mathrm{~A} / \mathrm{m}$, $\Delta \mathrm{T} / \Delta \mathrm{t}=10{ }^{\circ} \mathrm{C} / \mathrm{min}$. Cette courbe caractéristique met en évidence deux phénomènes importants :

- l'existence d'un minimum pour $\mathrm{T} \approx 250{ }^{\circ} \mathrm{C}$ qui sépare les comportements temporels de $\mu_{\mathrm{i}}$ le long des isothermes.

- l'accélération de cinétiques juste avant le point de Curie $\mathrm{T}_{\mathrm{C}}=415^{\circ} \mathrm{C}$

(pic d'Hopkinson [2]). 


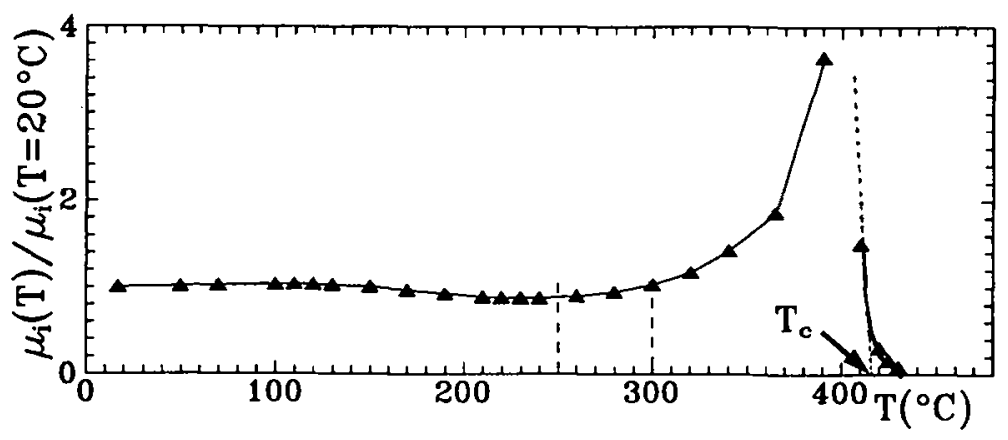

Fig. 2.- Evolution avec la température de la perméabilité initiale normaliséc.

A partir de cette caractéristique, on obtient les courbes des figures 3 a-b qui représentent les variations isothermes de $\mu_{\mathrm{i}}$ à différentes températures de recuit. La valeur et la fréquence du champ de mesure sont respectivement $0,1 \mathrm{~A} / \mathrm{m}$ et $1 \mathrm{kHz}$.
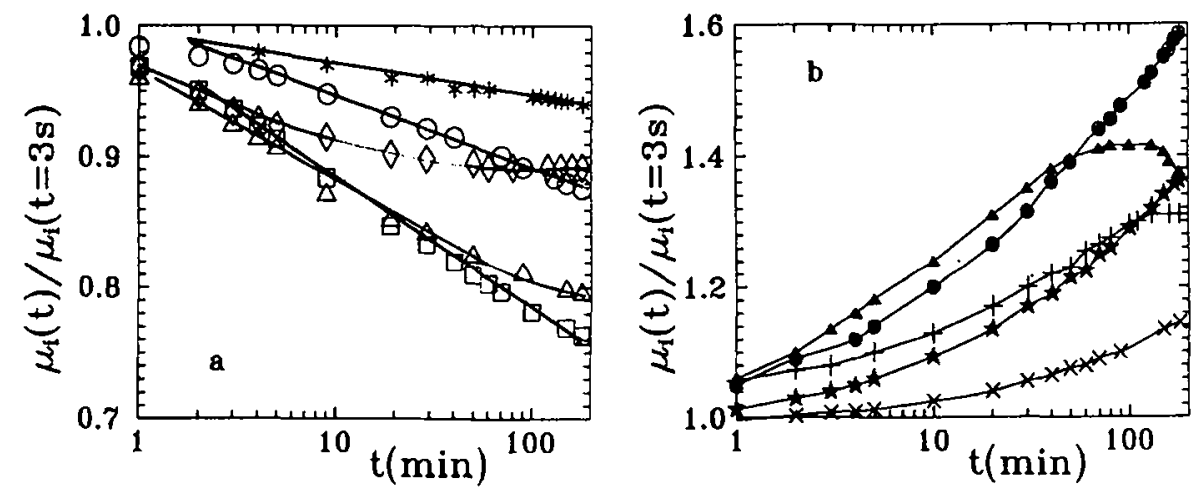

Fig. 3a-b :Evolutions isothermes de la perméabilité initiale à différentes températures : $* 20^{\circ} \mathrm{C}, \mathrm{O}^{\circ} 100^{\circ} \mathrm{C}$, ㅁ $150^{\circ} \mathrm{C}, \triangle 200^{\circ} \mathrm{C}, \diamond 250^{\circ} \mathrm{C}, \times 300^{\circ} \mathrm{C}, 340^{\circ} \mathrm{C}, \bullet 360^{\circ} \mathrm{C}, 380^{\circ} \mathrm{C}, \star 400^{\circ} \mathrm{C}$.

Pour des températures inférieures à $250^{\circ} \mathrm{C}$, toutes les courbes révèlent une dépendance décroissante et logarithmique dans un intervalle de temps dépendant de la température(Fig 3a). On peut considérer le modèle théorique correspondant à une distribution des constantes de temps constante entre deux limites $\tau_{1}$ et $\tau_{2}$ et nulle ailleurs ([3] [4] [6]). La loi logarithmique observée est en bon accord avec ce modèle si $\tau_{1} \ll t<\tau_{2}$. Ces variations sont liées à la création d'une anisotropie locale induite par la relaxation magnétique des atomes de Fer près du volume libre environnant. Il y a des phénomènes de "désaccommodation de la perméabilité initiale" correspondant à la stabilisation des parois de domaines autour de leur position d'équilibre aboutissant ainsi à la création d'un potentiel de stabilisation.

Les cinétiques montrées sur la figure $3 \mathrm{~b}$ sont régies par des phénomènes d'accommodation liés à la mobilité des parois qui s'accompagne de la relaxation des contraintes. On aboutit dans ce cas à une transformation irréversible du matériau (voir Figs. 1 et 6. a-b) . 
Nous avons effectué, à la température ambiante, des mesures de désaccommodation de la perméabilité initiale $\left(\Delta \mu_{\mathrm{i}} / \mu_{\mathrm{i}}\right)$ sur les divers échantillons recuits. Le champ de mesure est identique à celui utilisé lors des mesures en paliers isothermes. Le temps d'observation est de 3 min. après désaimantation. Le rapport $\Delta \mu_{\mathrm{i}} / \mu_{\mathrm{i}}$ est défini comme suit :

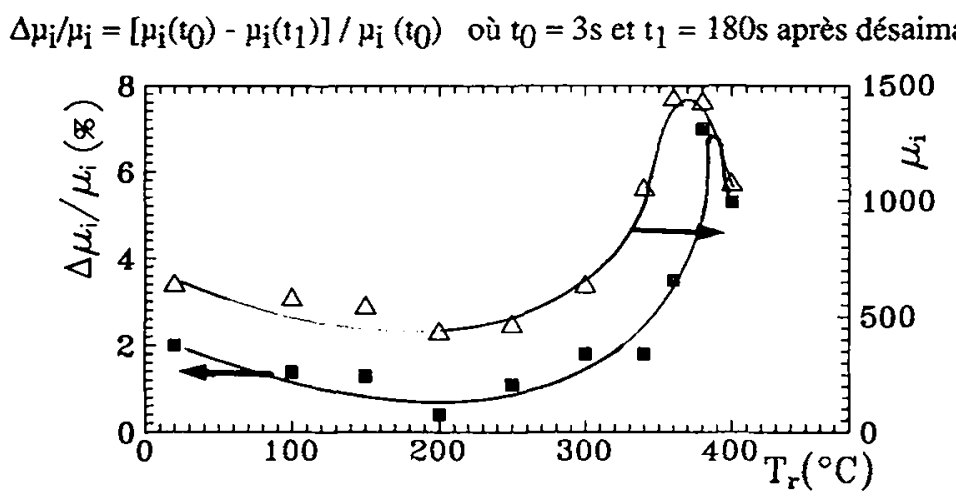

Fig. 4.- Variations à la température ambiante de $\mu_{i}$ et de $\Delta \mu_{i} / \mu_{i}$ en fonction de $T_{r}$.

Nous constatons que :

-- sur la figure 4 les deux évolutions sont similaires.

-- ces courbes correspondent bien à la caractéristique du matériau portée sur la figure 2.

Ces résultats traduisent une évolution de l'anisotropie magnétique induite, nous pouvons écrire [5] :

$$
(\Delta \mathrm{B} / \mathrm{B})_{\mathrm{B}=0}=\Delta \mu_{\mathrm{i}} / \mu_{\mathrm{i}}=\left(5 . \pi^{2} \cdot \mu_{0} \cdot \mathrm{L} \cdot \mathrm{K}_{\mathrm{u}}\right) /\left(8 \cdot \mathrm{A} \cdot \mathrm{B}_{\mathrm{s}}\right)
$$

où $\mathrm{L}, \mathrm{A}, \mathrm{B}_{\mathbf{S}}, \mathrm{K}_{\mathfrak{u}}$ sont respectivement la distance entre parois, la largeur des parois, l'induction à saturation et la constante d'anisotropie induite.

Etant donné que les recuits à basse température $\left(20-250^{\circ} \mathrm{C}\right)$ n'induisent pas de changement notable dans l'approche à saturation (Fig. 6a), les effets observés peuvent s'expliquer en termes de diminution de l'anisotropie locale dans le sens du ruban par rapport à l'état brut de trempe. Il est à noter que nous observons des structures en domaines sur l'échantillon brut de trempe visibles par effet Kerr sans polir l'échantillon. L'existence d'une telle structure permet d'expliquer la préexistence d'une anisotropie magnétique. Lors des recuits, la désorientation des moments magnétiques près du volume libre reste toutefois faible compte tenu de la complexité des structures en domaines et des gradients de contraintes.

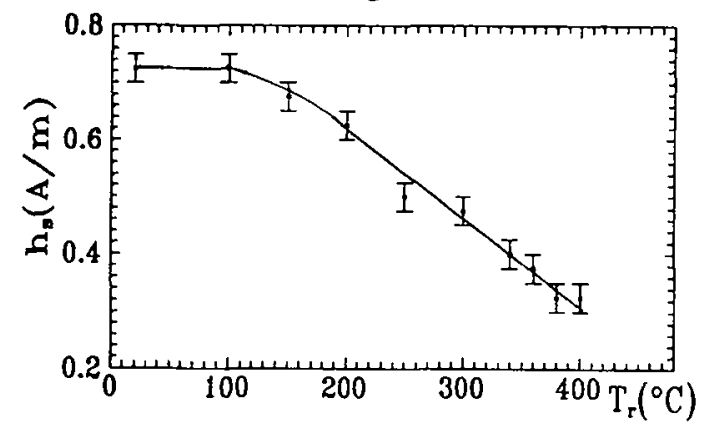

Fig.5.- Variation avec $T_{r}$ du champ de stabilisation pour des recuits de $3 \mathrm{~h}$. 
Un autre paramètre important caractérisant le comportement à faible champ est le champ de stabilisation (ou "pinning") $: h_{s}$. Il correspond au maximum de la courbe $(\Delta \mu / \mu)=f\left(h_{a c}\right)$. D'après la théorie de Chikazumi [6], les gradients des contraintes internes produits par la trempe sont à l'origine de l'ancrage des parois de domaines dans leur position d'équilibre . Le mouvement irréversible de ces parois intervient lorsque le champ d'excitation $h_{a c}$ excède le champ de stabilisation dont la valeur est : $h_{s}=\left(3 . \lambda_{s} \sigma . A\right) /\left(B_{s} .1\right)$ où $\lambda_{s}, \sigma, 1$ sont respectivement la constante de magnétostriction à saturation, la valeur moyenne des contraintes internes et la portée du champ de contraintes.

La relaxation des contraintes internes devrait réduire $h_{S}$. C'est ce que nous observons expérimentalement (Fig. 5) . Nous remarquons aussi une certaine analogie entre les courbes $h_{S}\left(T_{r}\right)$ et TRS $\left(T_{r}\right)$ à savoir que leur évolution n'intervient qu'à partir de $T_{r} \approx 150^{\circ} \mathrm{C}$.
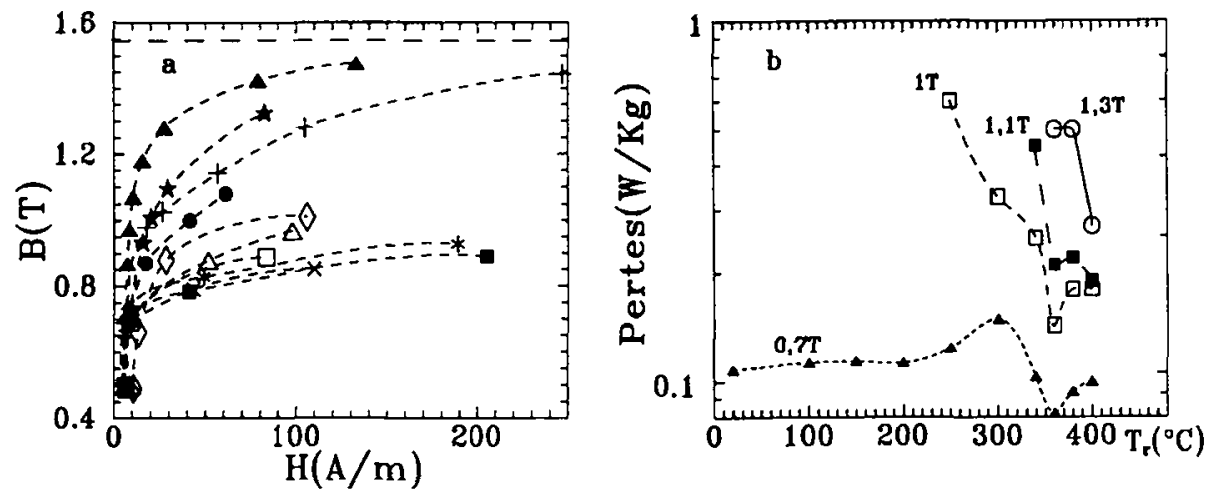

Fig. 6-a : Courbes de magnétisation à $50 \mathrm{~Hz}$ pour les recuits suivants : $\times 100^{\circ} \mathrm{C}$, $150^{\circ} \mathrm{C}, \square 200^{\circ} \mathrm{C}$, $\triangle 250^{\circ} \mathrm{C}, \diamond 300^{\circ} \mathrm{C}, \quad 340^{\circ} \mathrm{C},+360^{\circ} \mathrm{C}, \star 380^{\circ} \mathrm{C}, \triangle 400^{\circ} \mathrm{C}$ (duré $3 \mathrm{~h}$ ) et $*$ brut .

6-b : Pertes massiques à $50 \mathrm{~Hz}$ des différents échantillons recuits pour divers niveaux d'induction .

Sur les figures 6. a-b sont reportées des mesures magnétiques à $50 \mathrm{~Hz}$ dans les conditions de flux sinusoïdal. Les traitements thermiques, par le biais de la relaxation structurale, favorisent la création d'un certain ordre magnétique dans le sens de l'axe du ruban ce qui est en accord avec les considérations faites précédemment.

\section{Conclusions}

Le comportement à faible champ renseigne sur les évolutions de l'anisotropie magnétique locale $\left(\mu_{\mathrm{i}} \propto \mathrm{K}_{\mathrm{u}}\right)$ et la relaxation des contraintes internes $\left(h_{\mathrm{S}} \propto \sigma\right)$.

Nous avons observé, à partir des mesures le long des isothermes de la perméabilité initiale, qu'il existe dans l'alliage 2605 \$2 deux cinétiques opposées de la relaxation magnétique.

L'apparition d'un ferromagnétisme doux est liée à la mobilité des parois due à l'annihilation des gradients des contraintes internes (champs de "pinning") ceci grâce à des réarrangements structuraux de type topologique (T.S.R.O.).

[1] ALVES, F. et PERRON, J.C., à paraître dans J.Magn.Magn.Mater.(1992) .

[2] MIYAZAKI, T. ct TAKAHASHI, M. , $2^{\text {nd }}$ Symp. Strucl. Prop. Amorphous Metals, Aichi, Japan (1979).

[3] KROMMÜLLER, H. , Nachwirkung in Ferromagnetica, Springer-Verlag, (1968) .

[4] FÖLDEAKI, M. et al., Phil.Mag.B, Vol.B, (1991) 1101-1117.

[5] ALLIA, P. et VINAI, F. , IEEE Trans.Mag.MAG17, (1981) 1481.

[6] CHIKAZUMI, S. , Physics of Magnetism, J.Wilcy \& Sons Inc., (1964). 